\title{
Contents, Vol. 124, 1962
}

\section{Dermatologica}

Journal International de Dermatologie - International Journal of Dermatology - Dermatologische Zeitschrift

Gegründet von Oskar LassaГ (1893-1907) Weitergeführt von Erich Hofifmann (1908-1938)

Wilhelm Lutz (1939-1958)

OffizieUes Organ der Schweizerischen Gesellschaft für Dermatologie und Venereologie Organe officiel de la Société Suisse de Dermatologie et de Vénéréologie

Official Organ of the Nederlandse Vereniging van Dermatologen

EDITORES ET COLLABORATORES

J. R.Prakken, Amsterdam T. PUTKONEN, Helsinki M. I. QUIROGA, Buenos Aires F. E.

RABELLO, Rio de Janeiro

E. RAJKA, Budapest

J. Ramos e Silva, Rio de Janeiro

Th. Ravnáy, Szeged

H. Reçat Sigindim, Istanbul

F. REISS, New York

J. ROEDERER, Strasbourg J. ROKSTAD, Bergen M. RüITER, Groningen F. Sagher, Jerusalem

E. A. SAINZ DE AjA, Madrid

F. SCHAAF, Basel

W. SCHÖNFELD, Heidelberg H. SCHUERMANN, Bonn

H. W. Siemens, Leyden

C. E. SONCK, Turku

H. StORCK, Zurich

M. B. SULZBERGER, New York

L. SZODORAY, Debrecen

J. TAPPEINER, Wien

A. TOURAINE, Paris

M. TRUFFI, Pavia

L. VAN DER MeiREN, Bruxelle $\beta$

X. VILANOVA, Barcelona

A. WIEDMANN, Wien

G. S. Williamson, Ottawa

F. WORINGER, Strasbourg F. WORTMANN, Basel R. YAMAMOTO, Kyoto

W. JADASSOHN, Geneve - R. SCHUPPLI, Basel

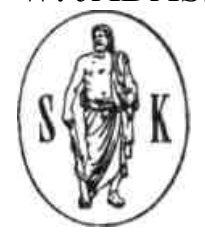


Vol. 124

1962

BASEL (Schweiz) S. KARGER NEW YORK

Alle Rechte, insbesondere das der Übersetzung in freuide Sprachen, vorbehalten

Ohne auadrückliche Genehmígung des Verlages ist es auch nicht gestattet, dieses Heft oder Teile daraus

auf photomechanischem Wege (Photokopie, Mikrokopie) zu vervielfaltigen.

Copyright 1962 by S. Karger AG, Basel Printed in Switzerland by Buchdruckerei Werner \&

Bischoff AG, Basel Cliches: Aberegg-Steiner \& Cie, AG, Bern, und Steiner \& Cie, AG, Basel

INDEX

Van Baak, J.: vide Van Delden, J.

Bandmann, H.-J.: Die Histologie doppelt exponierter Läppchenproben 205

Baumgartneb, P.: vide Kuske, $H$.

Beer, P.: $\quad$ Untersuchungen über die Wirkung der Vitamin-A-Säure 192

De Bergh, J.: Teleangiectasia Macularis Eruptiva Perstans 367

De Bergh, J. and Mali, J. W. H.:

Demonstrations 384

Berrens, L.: Chemical Characteristics of House Dust Allergen 372

Berzups, S.: Vide Storck, H.

Biberstein, H. H. und Jessner, M:

Weitere experimentelle Untersuchungen über das Shopepapillom (mit

Verwendung von Freunds Adjuvant) 1

Burckhardt, W.: Demonstrationen 261

Chaglassian, H. T.: vide Kurban, A. K.

Checiñski, T. and Maldyk, E.:

A Case of Mycosis Fungoides. Having a Feature of Malignant Prolife

ration of the Reticular Cells of the Skin and Internal Organs 420

Chorzelski, T: Experimentally Induced Acantholysis in Hailey's Benign Pemphigus 21

Cormane, R. H.: Comparative Enzyme-Histochemical Studies of the Human Skin (Nor

mal and Abnormal) 359

Demonstrations $\quad 382$

Cormane, R. H. and Vleugels Schutter, G. J. N.:

Demonstrations $\quad 382$

Debrunner, F.: Dermatofibrosarkoma protuberans. Bericht über 7 Fälle $\quad 341$

Delacrétaz, J. et Geiser, J. D.:

Demonstrations 276

Van Delden, J.: Demonstrations 382

Van Delden, J. and Van Baak, J.:

Demonstrations $\quad 383$

IV

Diem, E.; Jadassohn, W. et Paillard, R.:

Demonstrations 264

Van Dijk, E.: Demonstrations 376

Van Dijk, E. and De Jongh, J.:

Demonstrations $\quad 376$

Van Dijk, E. and Van Zuiden, E.: 
Demonstrations

376

Dirksen, H. J.: Demonstrations 380

Dirksen, H. J. and Driessen, F. M. L.:

Demonstrations $\quad 381$

Dirksen, H. J. and Stigter, W.:

Demonstrations $\quad 380$

Van Driel, L. M. J.: vide Young, E.

Driessen, F. M. L.: vide Dirksen, H. J.

Farah, F. S.: vide Kurban, A. K.

Franceschetti, A.; Klein, D.; Jadassohn, W. et Paillard, R.:

Demonstrations 263

Frenk, E.: $\quad$ Akute Putzmittelekzeme der Hände durch Tetrachlorsalicylanilid .. 433

Frey, J. R. und Geleick, H.:

Intranodale (intralymphatische) Injektion von Dinitrochlorbenzol

und Kontaktekzem des Meerschweinchens 321

Sensibilisierungsweg und Sensibilisierungserfolg beim Kontaktekzem

des Meerschweinchens durch Dinitrochlorbenzol 389

Ceiser, J. D.: Le lupus érythémateux disséminé chez Tenfant. Remarques sur sa fre

quence et sur son pronostic 129

Etude expérimentale de Faction antifongique de deux antiserotoniques 245

Geiser, J. D.: vide Delacrétaz, J.

Geleick, H.: vide Frey, J. R.

Götz, H. und Pascher, G.:

Über die chemische Zusammensetzung des Trichophyton mentagro-

phytes var. granulosum, T. schönleinii und T. rubrum im Vergleich

zu Schimmelpilzen (Penicillium, Cladosporium herbarum und Fusa-

rium) $\quad 31$

Golay, M.; Jadassohn, W. et Paillard, R.:

Demonstrations 264

Heite, H.-J. und Ritter, K.:

Fehlerkritische Studie zur Messung der Epidermisbreite beim Akan-

those-Test 406

Van Der Horst, J. N.: vide Waterman, A. H.

$\mathrm{V}$

Hunziker, N. et Schinas, G.:

Experiences sur cobayes nouveau-nés.

Trichophytie 231

Eczema au dinitrochlorobenzène 235

Jadassohn, W.: vide Diem, E.

Jadassohn, W.: vide Franceschetti, A.

Jadassohn, W.: vide Golay, M.

Jadassohn, W.: vide Mach, R. S.

Jadassohn, W. et Paillard, R.:

Demonstrations 265

Jansen, L. H.: Demonstrations

Jeanrenaud, B.: vide Mach, R. S. 
Jessner, M.: vide Biberstein, H. H.

De Jongh, J.: vide Van Dijk, E.

De Jongh, J.: vide Kalsbeek, G. L.

Jonkers, G. H.: Demonstrations

384

Jung, E. G. und Schnyder, U. W.:

Die «Erythrodermie ichthyosioforme congénitale»: ein heterogenes

Syndrom 189

Kalsbeek, G. L.: Demonstrations 381

Kalsbeek, G. L. and De Jongh, J.:

Demonstrations $\quad 381$

Katzenellenbogen, I.:

Lichen Planus Actinicus (Lichen Planus in Subtropical Countries) ..

Klein, D.: vide Franceschetti, A.

Krebs, A.: $\quad$ Zur Klinik und Therapie der Psoriasis arthropathica 249

Kurban, A. K.; Farah, F. S. and Chaglassian, H. T.:

Alopecia Mucinosa. A Histochemical Study 398

Kuske, H.; Baumgartner, P. und Mitarbeiter:

Demonstrationen 296

Locher, G.: Permeabilitätsprüfung der Haut Ekzemkranker und Hautgesunder

für den neuen Indikator Nitrazingelb «Geigy», Modifizierung der Alka-

liresistenzprobe, $\mathrm{pH}-$ Verlauf in der Tiefe des Stratum corneum 159

Lozeron, H.: vide Maggiora, A.

Luger, A.: vide Schmid, E. E.

Mach, R. S.; Jeanrenaud, B.; Jadassohn, W. et Paillard, R.:

Demonstrations 263

VI

Macheh, E. und Vogell, W.:

Elektronenmikroskopische Untersuchungen an Hautkapillaren $\quad 110$

Maggiora, A.: Sérotonine et effet local des rayons X.

2e communication. Sérotonine et effet des rayons X sur les mitoses du

bulbe pileux et Tacanthose radiologique 183

3e communication. De la durée de la protection due à la Sérotonine

vis-à-vis de $\Gamma$ effet épilateur chez le cobaye 385

Maggiora, A. et Lozeron, H.:

De Гeczéma experimental (16e communication). Diminution de la

reaction eczémateuse chez les cobayes irradiés au préalable 240

Maldyk, E.: vide Checiñski, T.

Mali, J. W. H.: vide De Bergh, J.

Mashkilleison, A. L.: vide Per, M. I.

Meyer, A. P. W. R.: vide Young, E.

Michalowski, R.: Nævus sébacé de Jadassohn - un état précancéreux 326

Müller, J.: Untersuchungen zur Prognose und Therapie der Mycosis fungoides .

Nilzén, Å. and Voss-Lagerlund, K.:

Epicutaneous Tests with Detergents and a Number of other Common

Allergens

42

Ossenkoppele, W. K.: 
Demonstrations

Paillard, R.: vide Diem, E.

Paillard, R.: vide Franceschetti, A.

Paillard, R.: vide Golay, M.

Paillard, R.: vide Jadassohn, W.

Paillard, R.: vide Mach, R. S.

Pascher, G.: vide Go $\tau$, $\mathrm{H}$.

Paschoud, J.-M.: Kritische Bemerkungen zur Zusammensetzung der sogenannten

Standardreihen für epikutane Testproben 196

Demonstrations 281

Per, M. I. and Mashkilleison, A. L.:

Remote Results of Continuous Systemic Corticosteroid Treatment

in Pemphigus 99

Ritter, K.: vide Heite, H.-J.

Rottier, P. B.: Improved Determination of Sensitivity for Ultraviolet Rays 356

Schinas, G.: vide Hunziker, N.

Schmid, E. E. und Luger, A.:

Serologische Untersuchungen bei endemischer Syphilis

VII

Schnyder, U. W.: vide Jung, E. G.

Schnyder, U. W.: vide Storck, H.

Schuppli, R.: Über die Bekämpfung der Syphylis bei Homosexuellen 155

Demonstrationen 270

Schwarz, K.: vide Storck, H.

Schwarz, K.: vide Sulser, H.

Van Steenbergen, E. P. and Vinks, P. A.:

Demonstrations $\quad 384$

Steimeyer, L. J. P.:

Demonstrations $\quad 382$

Stigter, W.: vide Dirksen, H. J.

Storck, H. und Berzups, S.:

Über Lupus erythematodes unter besonderer Berücksichtigung des

Uberganges von lokalisierten in generalisierte Formen 142

Storck, H.; Schnyder, U. W. und Schwarz, K.:

Demonstrationen 289

Stüttgen, G.: Zur Lokalbehandlung von Keratosen mit Vitamin-A-Säure

Sulser, H. und Schwarz, K.:

Das Verhalten der Erythrocyten-Katalase-Aktivität im Verlaufe der experimentellen Sensibilisierung mit p-Hydroxylaminobenzolsulfon-

säureamid beim Meerschweinchen 218

Vinks, P. A.: vide Van Steenbergen, E. P.

Vinks, P. A.: vide Waterman, A. H.

Vleugels Schutter, G. J. N.: vide Cohmane, R. H.

Vogell, W.: vide Macher, E.

Voss-Lagerlund, K.: vide Nilzén, A.

Waterman, A. H.: Demonstrations 
Waterman, A. H. and Van Der Horst, J. N.:

Demonstrations

379

Waterman, A. H. and Vinks, P. A.:

Demonstrations $\quad 380$

Young, E.: $\quad$ The Significance of House Dust Allergen 368

Young, E. and Van Driel, L. M. J.:

Demonstrations $\quad 377$

Younc, E. and Meyer, A. P. W. R.:

Demonstrations 377

Van Zuiden, E.: vide Van Dijk, E.

VIII

VERHANDLUNGSBERICHTE - PROCEEDINGS - CONGRÈS

161st Meeting of Netherlands Society of Dermatologists, May 27,

1961, Utrecht 355

43. Jahresversammlung der Schweizerischen Gesellschaft für Derma

tologie und Venereologie, 30. September und 1. Oktober 1961 in

Bern - 43e Reunion annuelle de la Société Suisse de Dermatologie et

de Venereologie, les 30 septembre et ler octobre 1961 à Berne 129

BUCHBESPRECHUNGEN - BOOK REVIEWS - LIVRES NOUVEAUX 436

VARIA $\quad 440$

INDEX RERUM 441 